\title{
Normal pressure hydrocephalus - active and passive pathogenic mechanisms
}

\author{
St.M. Iencean ${ }^{1}$, Al. Tascu ${ }^{2}$, A.St. Iencean ${ }^{3}$, I. Poeata ${ }^{1}$, M.R. Gorgan ${ }^{2}$ \\ 1"Grigore T. Popa" University of Medicine and Pharmacy Iasi, Romania \\ "Carol Davila" University of Medicine and Pharmacy Bucharest, Romania \\ 3“N. Oblu” Emergency Hospital Iasi, Romania
}

\begin{abstract}
Normal pressure hydrocephalus (NPH) is characterized by normal CSF pressure, less than $18 \mathrm{~cm} \mathrm{H} 2 \mathrm{O}$, classical clinical triad: gait disturbance, dementia and incontinence in patients with communicating hydrocephalus on $\mathrm{CT}$ or MRI.

We analyzed retrospectively the NPH hospitalized patients in three neurosurgical departments between July 2007 and December 2012. Only the cases who met all diagnostic criteria were selected for this study. There were 47 selected cases of patients with NPH, including 24 patients with secondary NPH and 23 patients with idiopathic NPH. Ventriculo-peritoneal shunt was performed in all 24 patients with secondary NPH and at 11 patients with IdNPH. The short-term and long-term results were good and very good for cases of secondary NPH and good in $60 \%$ and poor in $40 \%$ in cases of IdNPH.

The MR imaging showed the absence of CSF passage through the ventricular wall and the ventricular wall in cases of IdNPH with poor results after shunting: ependyma and glia limitans interna represents a fluid parenchymal barrier between the brain parenchyma and the ventricles as a glialependymal barrier.
\end{abstract}

We can consider that secondary NPH and some cases of idiopathic NPH with repeated small increases of ICP, with transependymal migration of CSF and hydrocephalus causing clinical triad because of the open glial-ependymal barrier, as an Active Normal Pressure Hydrocephalus and the shunt has good results. Other cases of IdNPH have not increases of intracranial pressure, no transependymal migration of CSF and there are periventricular deep lesions, without brain atrophy, causing clinical triad, as a passive hydrocephalus, it is a Passive Normal Pressure Hydrocephalus.

Key words: glial ependymal barrier, normal pressure hydrocephalus.

Normal pressure hydrocephalus (NPH) is characterized by normal CSF pressure, less than $18 \mathrm{~cm} \mathrm{H} 2 \mathrm{O}$, classical clinical triad: gait disturbance, dementia and incontinence in patients with communicating hydrocephalus on $\mathrm{CT}$ or MRI. Clinical triad has been explained on the basis of mechanical factors and ischemic factors. NPH probably represents the final common pathway for a number of different disease processes. Normal pressure hydrocephalus can be secondary in $50-70 \%$ and of unknown causes in $30-50$ 
$\%$, named idiopathic NPH (IdNPH. The diagnosis and management of idiopathic NPH remains unclear because the pathogenesis is unclear. In cases of secondary NPH the progressive ventricular enlargement follows of the decrease resorption of CSF with known cause and in IdNPH the progressive ventricular enlargement follows a transient increase in ICP, ventricles remain enlarged while ICP normalized. Many studies showed deep white matter ischemia and hypoperfusion dominant in the frontal areas and improvement after shunt surgery.

\section{Materials and methods}

We analyzed retrospectively the 47 $\mathrm{NPH}$ hospitalized patients in three neurosurgical departments between July 2007 and December 2011; there were 24 cases of secondary normal pressure hydrocephalus and 23 cases of idiopathic normal pressure hydrocephalus. History, clinics and the studies of MRI imaging and measurement of intracranial pressure allowed the diagnosis. Only the cases who met all diagnostic criteria were selected for this study. In 24 cases of secondary normal pressure hydrocephalus and in 13 cases of IdNPH we used ventriculo-peritoneal shunts with low or medium differentialpressure valve. Ten cases of idiopathic normal pressure hydrocephalus were not treated with ventriculo-peritoneal shunt. Postoperative follow-up included a monthly clinical evaluation and a computed tomographic (CT) scan or a MRI at 6 and 12 months after surgery.

\section{Results}

The CSF opening pressure in 24 cases of secondary NPH were between 8 to 17

$\mathrm{mm} \mathrm{Hg}$ and the clinical results after the ventriculo-peritoneal shunts were very good in 19 cases and good in 5 cases. In cases of idiopathic normal pressure hydrocephalus the CSF opening pressure was between 6 to $15 \mathrm{~mm} \mathrm{Hg}$. The results were : good in 6 cases with CSF-OP $=10$ - $15 \mathrm{~mm} \mathrm{Hg}$; poor results in 7 cases with $\mathrm{CSF}-\mathrm{OP}=7-13 \mathrm{~mm} \mathrm{Hg}$ and in 10 cases with $\mathrm{CSF}-\mathrm{OP}=6-12 \mathrm{~mm} \mathrm{Hg}$ no shunts. Figures 1 and 2 show the selected brain CT or MR images of these situations.

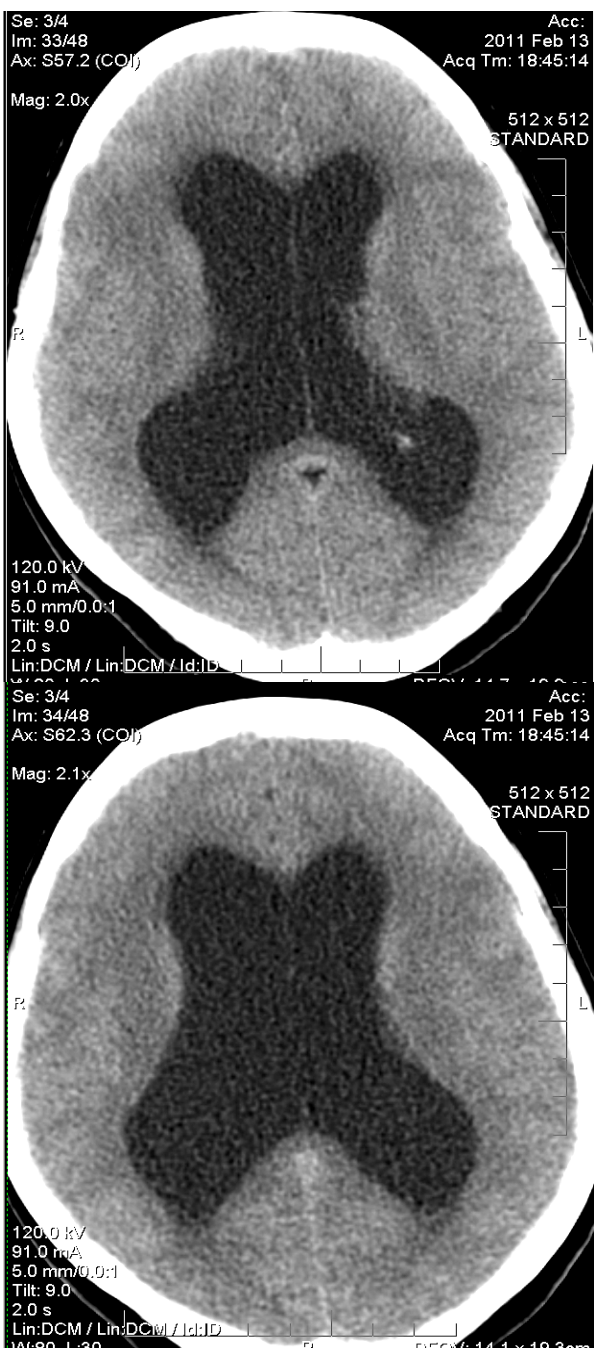

Figure 1 A

CT image of secondary NPH 


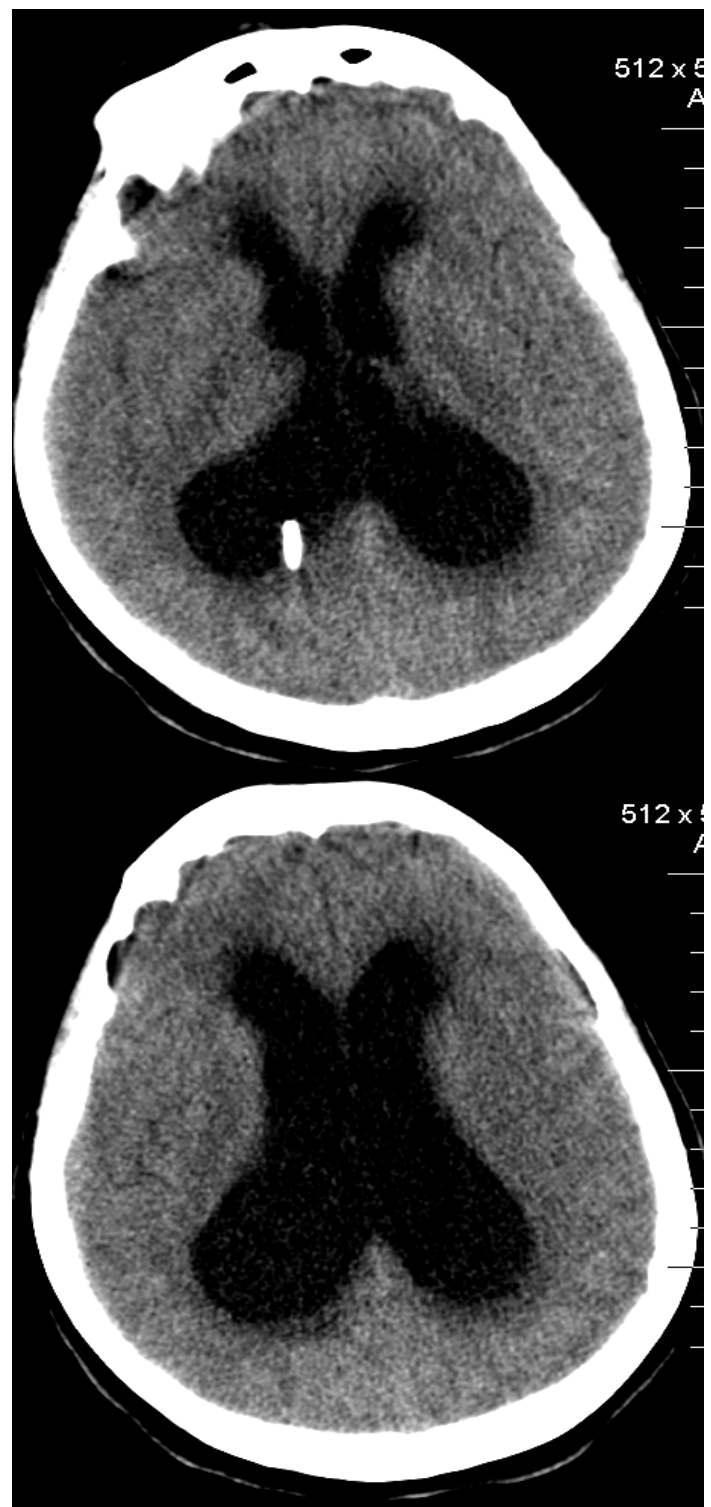

Figure $1 \mathrm{~B}$

CT image of secondary NPH after shunt with a very good clinical result

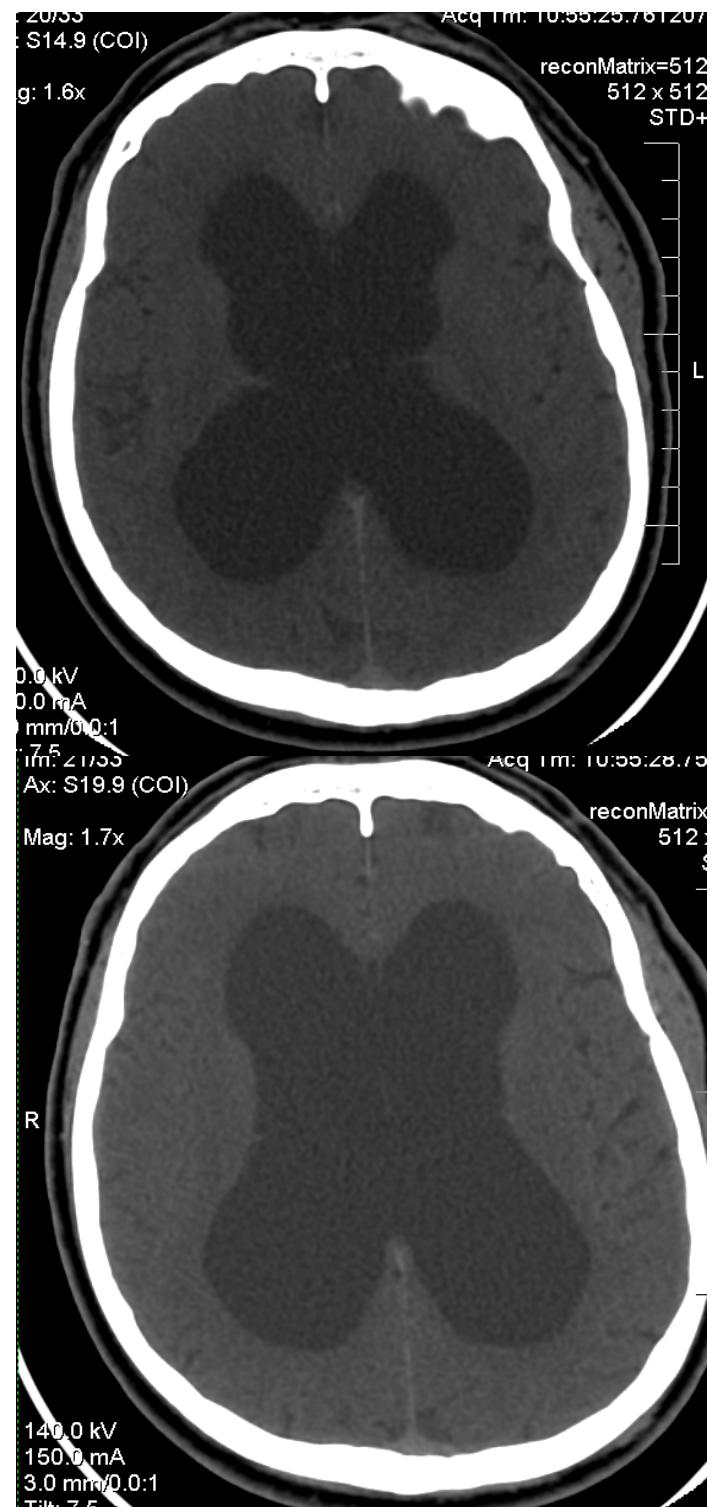

Figure $2 \mathrm{~A}$

$\mathrm{CT}$ images of idiopathic normal pressure hydrocephalus, $\mathrm{CSF}$ - $\mathrm{OP}=9 \mathrm{~mm} \mathrm{Hg}$ 


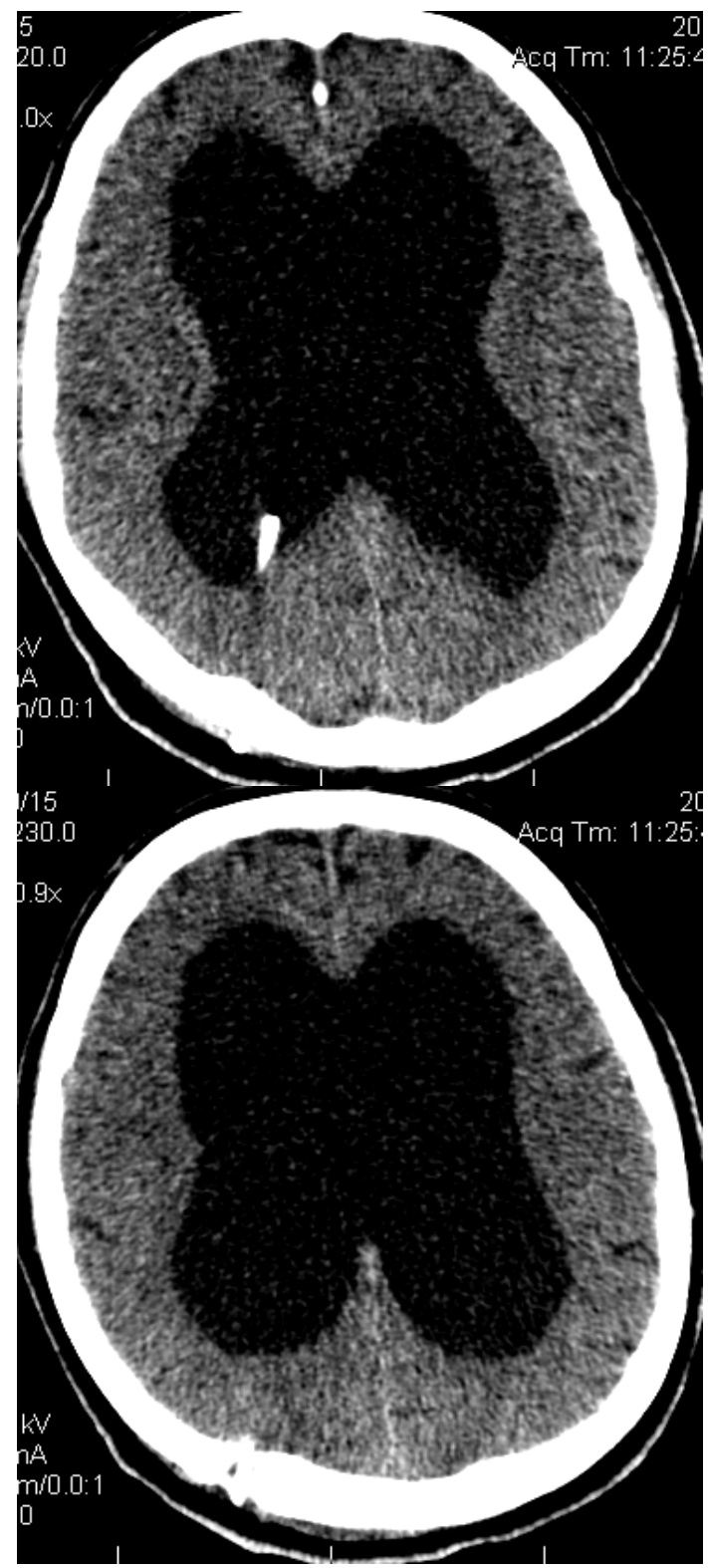

Figure 2 B

The same case after shunt, but poor result

\section{Discussions}

The diagnosis and management of NPH remains unclear because the pathogenesis is unclear. In normal pressure hydrocephalus the progressive ventricular enlargement follows of the decrease resorption or absorbtion of CSF with known cause in secondary NPH and unknown cause in
IdNPH and the progressive ventricular enlargement follows a transient increase in ICP, and the ventricles remain enlarged while ICP normalized.

Relkin N, Marmarou A et all. in 2005 established diagnostic criteria for idiopathic $\mathrm{NPH}$ and these are shown below:

\section{Clinical history:}

a. Insidious onset (versus acute)

b. Origin after age $40 \mathrm{yr}$

c. A minimum duration of at least 3 to $6 \mathrm{mo}$

d. No evidence of an antecedent event such as head trauma, intracerebral

hemorrhage, meningitis, or other known causes of secondary hydrocephalus

e. Progression over time

$f$. No other neurological, psychiatric, or general medical conditions that are sufficient to explain the presenting symptoms

\section{Brain imaging}

a. Ventricular enlargement not entirely attributable to cerebral atrophy or congenital enlargement (Evan's index 0.3 or comparable measure)

b. No macroscopic obstruction to CSF flow

c. At least one of the following supportive features

1. Enlargement of the temporal horns of the lateral ventricles not

entirely attributable to hippocampus atrophy

2. Callosal angle of 40 degrees or more

3. Evidence of altered brain water content, including periventricular signal changes on CT and MRI not attributable to microvascular ischemic changes or demyelination

4. An aqueductal or fourth ventricular flow void on MRI

\section{Clinical}

\section{Gait/balance disturbance}

a. Decreased step height

b. Decreased step length

c. Decreased cadence (speed of walking)

d. Increased trunk sway during walking 
e. Widened standing base

$f$. Toes turned outward on walking

g. Retropulsion (spontaneous or provoked)

h. En bloc turning (turning requiring three or more steps for 180 degrees)

$i$. Impaired walking balance, as evidenced by two or more corrections out of eight steps on tandem gait testing

Cognition - evidence of at least two of the following

a. Psychomotor slowing (increased response latency)

b. Decreased fine motor speed

c. Decreased fine motor accuracy

d. Difficulty dividing or maintaining attention

e. Impaired recall, especially for recent events

f. Executive dysfunction, such as impairment in multistep procedures, working memory, formulation of abstractions/similarities, insight

g. Behavioral or personality changes

Urinary continence

a. Episodic or persistent urinary incontinence not attributable to primary

urological disorders

b. Persistent urinary incontinence

c. Urinary and fecal incontinence

or any two of the following

a. Urinary urgency as defined by frequent perception of a pressing need to void

b. Urinary frequency as defined by more than six voiding episodes in an average

12-hour period despite normal fluid intake

c. Nocturia as defined by the need to urinate more than two times in an average night

\section{Physiological}

CSF opening pressure in the range of 5$18 \mathrm{~mm} \mathrm{Hg}$ (or 70-245 mm H2O)

All our 47 cases met the criteria for inclusion in the group of normal pressure hydrocephalus.

Analysis of the cases showed that there were good results after shunt in all secondary normal pressure hydrocephalus with all clinical criteria, with CSF - OP between 9 to $17 \mathrm{~mm} \mathrm{Hg}$ and on brain imaging there was transependymal migration of CSF. Also there were good results in cases of idiopathic normal pressure hydrocephalus with all clinical criteria, with cerebro-spinal fluid pressure : $10-15 \mathrm{~mm} \mathrm{Hg}$. But there were poor results in cases of idiopathic normal pressure hydrocephalus with partial criteria, with CSF - OP $=6-13 \mathrm{~mm} \mathrm{Hg}$ and without transependymal migration of CSF on brain imaging. Also the cases with low CSF pressure have not been treated with drainage, with poor results.

Therefore good results have been after drainage in the cases with CSF-OP : $9-17$ $\mathrm{mm} \mathrm{Hg}$ and transependymal migration of CSF, with the ventricular walls permeable. It seems the ventricular wall consists of the glia limitans interna and the ependymal wall, is a glial-ependymal barrier or a fluidglial barrier and in these cases this barrier was open.

There were poor results in the cases with CSF - OP: 6 - $13 \mathrm{~mm} \mathrm{Hg}$ and without transependymal migration of CSF, as a closed fluid-glial barrier. These are cases of IdNPH with hydrocephalus, but they have not increase of CSF - OP, no transependymal migration of CSF as a closed fluid glial barrier and the drainage is not effective.

Internal hydrocephalus may occur by decreasing or blocking CSF absorption. Normal pressure hydrocephalus seems to be a particular situation of the internal hydrocephalus caused by blockage of CSF absorbtion and the increase of intracranial pressure ( CSF - OP) is small and repeted, without exceeding the normal limits. Secondary NPH has a known etiology: subarachnoid hemorrhage, meningitis etc. 
and Id NPH has the same mechanism of developing for hydrocephalus, but unknown etiology for the blockage of CSF absorbtion.

In cases of secondary or idiopathic $\mathrm{NPH}$, produced by blocking of the absorption of CSF, hydrocephalus occurs by increasing of CSF pressure (intracranial pressure $=$ ICP). These increases of intraventricular pressure are small and repeated, without exceeding the normal values of ICP and they cause clinical symptoms, therefore the ventricular drainage with low pressure valve is effective.

Passive developed hydrocephalus consists in diminution of brain parenchyma with passive replacement by CSF of such increased ventricular space. Situations with a progressive deep decrease of the periventricular cerebral parenchyma with slow and passive growth of the ventricular dimensions may correspond to cases of normal pressure hydrocephalus in which the ventricular drainage is not effective and which are commonly included in IdNPH. Therefore in these cases clinical triad results of periventricular parenchymal lesions and the ventricular drainage is not effective.

It seems that normal pressure hydrocephalus is a communicating hydrocephalus with two types:

- NPH with open fluid glial barrier when hydrocephalus causing the clinical triad and the shunt is effective, and

- NPH with closed fluid glial barrier, when the periventricular deep lesions causing the clinical triad, as a passive hydrocephalus and the ventricular drainage is not effective.

Therefore normal pressure hydrocephalus with known or unknown etiology, secondary or idiopathic NPH, may present clinically with an open fluidglial barrier, with repeated small increases of ICP and transependymal CSF and hydrocephalus causes clinics as an active normal pressure hydrocephalus with effective ventricular drainage. This is a form of the internal hydrocephalus when the increases of intraventricular pressure are small and repeted without exceeding normal values of ICP.

The second clinical form is a normal pressure hydrocephalus with a closed fluidglial barrier, without increases of ICP and no transependymal CSF without brain atrophy but the periventricular deep lesions causing clinics as a passive hydrocephalus and the ventricular drainage is not effective. This is a form of passive hydrocephalus without brain atrophy, but with deep periventricular parenchyma lesions.

There are two different pathogenic mechanisms for these two different clinical forms of normal pressure hydrocephalus, but historically they have been included in the same group because of similar characteristics; also the different therapeutic efficiency of the same traitment is an argument for this differentiation.

\section{Conclusions}

Diagnosis criteria are very important in normal pressure hydrocephalus.

We propose a differentiation of NPH whatever the etiology, known or unknown of the blockage of CSF absorbtion, but in relation to the clinical pathogenic mechanisms. An active normal pressure hydrocephalus, secondary or idiopathic form, in which there are repeated small increases of ICP with transependymal CSF and hydrocephalus causes clinics and the 
ventricular drainage is effective. A passive normal pressure hydrocephalus has hydrocephalus, but not increases of intraventricular pressure and the clinic triad is caused of the deep periventricular lesions, without brain atrophy, and the ventricular drainage is not effective.

\section{Correspondence:}

Tascu Alexandru

" Carol Davila" University of Medicine and

Pharmacy Bucharest, Romania

E-mail:tascu_alexandru@yahoo.com

\section{References}

1. Adams RD, Fisher CM, Hakim S, et al. Symptomatic occult hydrocephalus with "normal" cerebrospinal pressures: a treatable syndrome. $\quad \mathrm{N}$ Engl J Med 1965;273:117;

2. McHugh PR. Occult hydrocephalus. Q J Med 1964;33:297-308;

3. Bradley WG Normal Pressure hydrocepohalus:
New Concepts on Etiology and Diagnosis. Commentary. AJNR. 21 Oct 2000;

4. U. Meier Gravity valves for idiopathic normalpressure hydrocephalus: a prospective study with 60 patients Acta Neurochirurgica Supplementum, Volume 95, 2005, pp 201-205;

5. Iencean StM. Simultaneous hypersecretion of CSF and of brain interstitial fluid causes idiopathic intracranial hypertension. Med Hypotheses. 2003 ;61(56):529-32;

6. Iencean StM. Idiopathic intracranial hypertension and idiopathic normal pressure hydrocephalus: diseases with opposite pathogenesis?Med Hypotheses. 2003;61(5-6):526-8;

7. Relkin N, Marmarou A, Klinge P, Bergsneider M, Black PM. Diagnosing idiopathic normal-pressure hydrocephalus. Neurosurgery. 2005 ;57 (3 Suppl):S416 ;

8. Iencean StM, Ciurea AV. Intracranial hypertension: classification and patterns of evolution. J Med Life. 2008;1(2):101-7;

9. Kiefer M, Unterberg A. The differential diagnosis and treatment of normal-pressure hydrocephalus. Dtsch Arztebl Int. 2012 ;109(1-2):15-25;

10. Verny M, Berrut G. Diagnosis of normal pressure hydrocephalus in elderly patients: a review. Geriatr Psychol Neuropsychiatr Vieil. $2012 ; 10(4): 415-425$. 